\title{
Bericht über die 63. Jahres-Versammlung der British Medical Association in London. 1895. ${ }^{{ }^{\prime}}$
}

\section{Section of Dermatology.}

\author{
A. Discussion on the Pathology and Treatment of Pru- \\ ritus. \\ I. Me Call Anderson erörtert zunächst die Frage, welche
} Theile des nervösen Apparates der Haut speciell das Gefühl des Juckens erzeugen, wenn sie gereizt werden. Es sind dies 1. die freien Nervenendigungen zwischen den Epidermiszellen oder in den Langerhansschen Zellen, 2. in den tieferen Lagen der Epidermis die Tastkörperchen, 3. der Bulbus vieler Haare. Im F'olgenden beschränkt sich Vortragender auf die Form des Hautjuckens, die gewöhnlich als Pruritus bezeichnet wird, bei der also das Jucken das einzige Hautsymptom ist. Pruritus ist die Folge einer directen, vielleicht bisweilen auch reflectorischen Störung der Hautnerven, unabhängig von irgend einer Reizquelle auf der Oberfläche der Haut. - Einige der hauptsächlichsten Ursachen des Pruritus werden besprochen: Der Pruritus des höheren Lebensalters hat seine Ursache in dem Mangel an Bewegung und in der Abnahme der functionellen Thätigkeit der Organe und Gewebe des Körpers, wodurch die Ernährung herabgesetzt wird und ein unreines und in Folge dessen reizendes Blut circulirt. Das $\boldsymbol{J}$ ucken bei I cterus ist zweifellos auf die Anwesenheit von Gallensäuren im Blute zu schieben; nicht so leicht zu erklären ist aber, weshalb viele Fälle von Gelbsucht schwersten Grades frei von Jucken sind, während leichtere Fälle zu schwerstem Pruritus Anlass geben. Viellejcht ist bei manchen Leuten der nervöse Apparat empfindlicher oder die Zusammensetzung der Galle hat in den einzelnen Fällen kleine Unterschiede, die sie mehr oder weniger irritirend machen. Functionelle und organische Erkrankungen der Geschlechtsund Harnorgane und Schwangerschaft sind wohlbekannte ätiologische Factoren des Pruritus, und die gichtische Diathese und Störungen der Verdauugsorgane sind die häufigsten aller Ursachen. Nicht so allgemein bekannt ist die Beziehung zwischen Diabetes mellitus und Pruritus vulvae bei älteren Frauen, zumal hier oft die typischen Symptome des Diabetes fehlen. Eine andere in-

1) The Brith. Med. Journ. 30. Nov. et 21. Dee. 1895. 
teressante Form ist der Pruritus hiemalis. Nach Diakonoff besteht hier primär eine abnorme Reizung der Hautgefühlsnerven, die reflectorisch eine localisirte paralytische Dilatation der Hautcapillaren veranlassen, wodurch die Ernährung der Gefühlsnerven gestört wird. Endlich kann die Krankheit Folge einer Geistesstörung sein.

Was die Behandlung angeht, so sind die örtlichen Mittel nur von vorübergehendem Nutzen. Ist Verdacht vorhanden, dass nervöse Schwäche oder solche der Ernährung besteht, so müssen Nerventonica versucht werden, z. B. Posphor, Arsen oder Strychnin, allein oder combinirt. Bulkley empfiehlt sehr Tinctura Gelsemini halbstündlich 10 Tropfen, oder bis zu einer Drachme in 2 Stunden, ausserdem Tinctura Cannabis 3mal täglich 10-30 Tropfen nach dem Essen und gut verdünnt. Vortragender hatte die besten Resultate mit A tropin, das er subcutan gibt; er beginnt mit $1 / 100$ Gran Abends und steigert die Dosis vorsichtig so lange, bis die physiologischen Wirkungen des Mittels auftreten und bis der Pruritus vollständig beseitigt ist. Ausserdem empfiehlt er Antipyrin und Phenacetin, bei denen die Dosis auch gesteigert werden muss, so dass schliesslich sehr hobe Dosen gat vertragen und mit Vortheil gebraucht werden. Sodann kann man auch die Elektricität in der Form des elektrischen Bades oder als constanten Strom mässiger Stärke Abends und Morgens je 10 Minuten anwenden und wenn das Jucken sehr störend ist, kann man als positiven Pol eine Schwammelektrode auf das obere Ende bringen und den anderen Pol ituf den untern Theil der Wirbelsäule.

II. H. A. G. Brooke. Pruritus in its relation to diseases of the skin.

Brooke erwähnt die Abhandlungen von Bronson (siehe Ref. in diesem Archiv 1892, Bd. XXIV, Seite 665), der das Hautjucken zu erklären sucht, als Perversionen des Gefühles, als Dysästhesie der Gefühlsnerven. Brooke selbst theilt die verschiedenen Fälle von Hautjucken in 2 Hauptclassen ein. In die erste bringt er alle Fälle, die nur durch Wirkung äusserer Ursachen entstehen, in die zweite solche, die ihren Ursprung rein internen Zuständen verdanken. In die zweite Gruppe gehören 1. solche Fälle von Pruritus, die einen nervösen Ursprung haben und diese können wieder in 2 Unterabtheilungen eingetheilt werden, je nachdem a) es sich um eine reine Erkrankung der Hautnerven handelt oder b) das Jucken secundär durch den Reiz einer nervösen Hauterkrankung oder reflectorisch durch andere Ursachen hervorgerufen wird.

a) Unter den rein nervösen Affectionen treffen wir die Classe pruriginöser Frkrankungen, die von den französischen Dermatologen, besondes B roc q, Besnier, Vidal und Leloir so fleissig studirt sind, bei denen der I'ruritas wesentlich die primäre Krankheit ist. Vortragender kann sich nicht allen Ansichten jener Autoren anschliessen; einige Fälle, die jene als primäre pruriginöse Neurodermatitis auffassen, scheinen ihm primäre Epidermiserkrankungen, andere durch im Blute circulirende Reizstoffe, hervorgerufen $z u$ sein. Brocq und Jacquet haben sich aber ein 
grosses Verdienst erworben, indem sie zeigten, dass ein primärer Pruritus durch das beständige Kratzen, zu dem er veranlasst, zu jener mehr oder weniger indurirten, papulösen, braun gefärbten Haut führen kann, die sie als lichenificirt bezeichnen. Früher hielt man diese Lichenification für ein chronisches Eczem oder einen Lichen und hielt sie eher für eine deutliche Localerkrankung, als für ein nur secundäres Symptom der allgemeinen Nervenreizbarkeit, der sie doch ihre Entstehung verdankt. - Nicht alle Fälle primären, nervösen Pruritus führen zur Entwickelung der Lichenification, so z. B. der Pruritus senilis; man hat sie lange bezeichnet als "pruritus sine prurigine". Solche Fälle können aber auch bei jungen Leuten und Kindern vorkommen, oder sie können auf umschriebene Stellen wie Gesicht, Hände, Znuge, Anus oder Genitalien beschränkt sein. Von diesen localen Varietäten rein nervösen Pruritus ist der des Anus und der Genitalien am besten bekannt; es werden aber auch Fälle hieher gerechnet, die, wenn auch zweifellos nervösen Ursprungs, doch nicht recht in diese Classe gehören: es handelt sich um die Fälle, bei denen die anscheinend normale Haut einen oder mehrere hyperästhetische Punkte aufweist, von denen das Jucken ausstrahlt, wenn sie durch Temperaturveränderungen, Schwankungen des Biutdruckes oder durch die Circulation toxischer Substanzen gereizt werden, welch' letztere im Körper entstanden oder von den Nahrungsmitteln abgespalten sein können. Solche Fälle unterscheiden sich vom echten nervösen Pruritus daảurch, dass die Zerstörung der hyperästhetischen Punkte, z. B. mit dem Galvanokauter, den ganzen Symptomencomplex zum Verschwinden bringt. - In diese Classe gehören auch andere Affectionen, bei denen das Jucken dem Auftreten irgend eines Ausschlages vorangeht, einige Eczeme und die Formen des "acuten lichen simplex", die Vidal und Besnier als urticariaähnlich angesehen haben, die aber besser unter den Begriff "papulöses Eczem" eingeschlossen werden. Die Prurigo von Hebra ist das am besten individualisirte Glied dieser Familie. - Besnier hat unter dem Titel „Diathetische Prurigo" eine ganze Familie dieser chronischen, polymorphen, pruriginösen Dermatitiden gruppirt, die in successiven Schüben wiederkehren und einen hauptsächlich eczemato-lichenoiden Charakter annehmen. Diese Affection kann vorübergehend oder dauernd die Haut freilassen, wofür dann Localisationen in den inneren Organen als: Emphysem, Asthma bronchiale, gastro-intestinale Störungen etc. auftreten. Dieser ganze Zustand kann hereditär sein, und zeigt sogar deutliche Zeichen von Atavismus. In diese Gruppe nervöser, juckender Krankheiten müssen auch jene prưiginösen Ausschläge papulösen oder vesiculösen, pemphigoiden Charakters eingereillt werden, die Tilbury Fox unter dem Namen Hydroa herpetiformis und Duhring als Dermatitis herpetiformis zusammenfasste. Rein nervöse Urticaria ist eine gut bekannte Affection; oft genügt schon der Gedanke, um einen Ausbruch von Quaddeln hervorzurufen mit dem dieselben begleitenden, intensiven Pruritus. - Jucken ist auch ein prämonitorisches Symptom einiger 
schwererer Nervenkrankheiten. Leloir hat mehrere dieser symptomatischen Prurigo unter dem Titel „Dermatoneuroses indicabrices" katalogisirt. Sie sind jedoch als Prodromina nicht sehr bäufig. Endlich gehören hierher die von Crodker als "Pruritus mentis“ bezeichneten Fälle, bei denen die Kranken sich unaufhörlich kratzen, da sie von dem Gedanken beherrscht werden, dass sie von Insecten gequält würdën oder dass Sandkörner oder dergleichen unter ibrer Haut lägen. Es handelt sich hier um eine Monomanie, und der Zustand ist eine hoffnungslose Form von Geistesstörung. - Die rein nervöse reflectorische Form des Juckens findet sich zeitweilig bei Jedermann. Selbst wenn man ganz frei von irgend welcher Hauterkrankung ist, bemerkt man irgendwo am Körper ein leichtes Jucken, worauf unmittelbar auch eine ähnliche Empfindung an anderen Körperstellen auftritt. Die Ursache ist nicht leicht aufzufinden; es ist möglicherweise nur eine vorübergehende, locale Reizung.

b) Die Fälle secundären nervösen Pruritus können zweckmässigerweise in 2 Classen eingetheilt werden, je nachdem sie

1. in Beziehung stehen zu Erkrankungen anderer Organe als der Haut, oder die Folge eines abnormen Reizes mechanischer oder physischer Art sind. In die erste Classe gehören die Zustände mehr oder weniger allgemein ausgebreiteten Juckens, die die Entwicklung ernster gastrischer oder intestinaler Erkrankungen, z. B. Carcinom begleiten oder ibr vorangehen, oder wie sie bei verschiedenen Krankheiten des Uterus auftreten. Man könnte hier an eine Art von Vergiftung denken, da aber ähnliches Jucken auch bei normaler Schwangerschaft und normaler Menstruation auftritt, muss man die Möglichkeit eines rein reflectorischen nervösen Ursprunges zulassen. - Bandwürmer verursachen durch mechanische Reizung oft intensivsten, allgemeinen Pruritus, der mit deren Beseitigung sofort schwindet. Einige Nahrungsmittel wirken offenbar anf dieselbe Art und Weise, z. B. verursacht Hafermehlbrei starken Hautreiz bei manchen Leuten, wenn er nach der gewöhnlichen schnellen Zubereitungs methode hergestellt wird, ist aber ganz unschuldig, wenn er gekocht wird, bis alle groben Partikel verschwunden sind. Das reflectorische Jucken in der Spitze des Penis bei Blasenstein und in der Nase, wenn Ascariden sich im Rectum aufhalten, sind bekannte Beispiele desselben Charakters. - In dasselbe Capitel gehört das durch Kälte- oder Hitzeeinwirkung erzeugte Jucken.

2. eine sehr bestimmte und in leichteren Entwicklungsgraden häufige Classe von Fällen bilden die, bei denen Pruritus entsteht durch den Reiz, den toxische Substanzen erzeugen, die im Körper gebildet und mit dem Blutstrom verbreitet wurden. Die bekanntesten Beispiele bilden das Jucken bei Diabetes, Gicht, Rheumatismus, Nierenkrankheiten und Gelbsucht. Beim Icterus kann das Jucken der Ablagerung des Gallenpigments in der Haut vorangehen, kann daher nicht der Ablagerung und directen Reizung der Gallenproducte selbst zugeschrieben werden. Pruritus ani findet sich oft bei Gicht und verschwindet bisweilen beim Auf- 
treten eines acuten Gichtanfalles. Allgemeines Jucken bildet eines der vielfachen Symptome träger Leberaction, die Murchison Lithaemie nannte. Der Pruritus bei Obstipation und träger Verdauung ist von derselben Art. - Der urticarielle Pruritus der Kinder findet sich häufig bei Rhachitis and wird durch antirhachitische Behandlung geheilt. Besnier nimmt an, dass manche Fälle von Altersjucken nicht auf mangelhafter Wirkung oder Degeneration der Nerven beruhen, sondern auf mangelhafter Arbeit der Nieren, wozu noch die schwache Herzaction als unterstïtzender Factor kommt.

3. Bei allen diesen Fällen ist das Auftreten des Juckens an eine gewisse Idiosynkrasie gebunden, denn es tritt nur bei einem kleinen Bruchtheil der an diesen Krankheiten Leidenden auf. Dies ist auch der Fall bei dem Jucken, das gewisse Nahrungsmittel und Arzneien erzeugen z. B. Schellfisch, Thee oder Kaffee, Alkohol, oder Opium, Belladonna, Quecksilber und Jodoform.

4. Der Einfluss des stärkeren Blutdrucks auf das Hervorbringen des Juckens beruht anscheinend auf dem mechanischen Druck auf die Nerven; das Jucken der Hämorrhoiden z. B. verschwindet, sobald der Druck beseitigt wird. Aehnlich ist wohl auch der Ano-genitale Pruritus der Schwangerschaft durch Anschwellen der Venenplexus des Beckens zu erklären.

5. Die Haut sonst gesunder Personen ist zeitweilig zu Jucken disponirt, weil die normale Geschmeidigkeit fehlt. Dafür hat man $z$ wei Erklärungen aufgestellt, nämlich dass dies Jucken entstünde durch Bildung kleiner Fissuren in den äusseren Lagen der Epidermis und dadurch theilweisem Freiliegen der epithelialeu Nervenendigungen oder dass es dem Einstellen der Absonderungsthätigkeit der Haut zuzuschreiben sei und der dadurch bedingten Reizung durch die nun nicht eliminirten, verbrauchten Producte des Blutes. Verf. möchte sich mehr der ersten Theorie anschliessen, da für gewöhnlich - ausgenommen solche Zustände wie Urämie und senile Atrophie - die Nieren genügen, um diese Elimination zu übernehmen.

Die verschiedenen äusseren Ursachen, die das Gefühl des Juckens hervorrufen, können etwa in 3 Classen eingetheilt werden, nämlich locale Hautkrankheiten, epizoische Parasiten und Reize physischer oder chemischer Natur. Vortragender erinnert daran, dass mauche Leute nach Berührung einer kleinsten Stelle der Haut mit Jodoform oder mit gewissen Pflanzen aus der Primulafamilie ein universelles Eczem bekommen und glaubt, dass auch Ekzeme parasitischen Ursprungs oder Eczeme, die von Parasiten besiedelt worden sind, in ähnlicher Weise den ganzen Körper ergreifen können, da von den Parasiten möglicherweise reizende Substanzen gebildet und dann von dem einzelnen Herde aus absorbirt und verbreitet würden. Dies ist therapeutisch wichtig, damit man nicht ein solches Eczem als constitutionell behandelt und den eigentlichen Herd der Reizung übersieht. Es erklärt auch die ausserordentliche Thatsache, 
dass eine so grosse Zahl der antipruritischen Mittel der Classe der Desinficientien und Bactericidien angehören.

Die reinen Pilzerkrankungen der Hant, tinea trichophytina und Pityriasis versicolor verursachen selten stärkeres Jucken; höchstens die letztere ausnahmsweise.

Die Wirkung der epizoischen Parasiten ist ja bekanntlich theils durch Einbringen von Secreten dieser Thiere, theils dnreh den Biss oder das Kriechen derselben oder auf beide Arten hervorgerufen. Unter den physikalischen Ursachen ist die bekannteste derplötzliche Temperaturwechsel. Derselbe scheint durch die schnelle Veränderung des Blutdrucks zu wirken. Dass heisses Wasser den Pruritus lindert erklärt sich vielleicht dadurch, dass es den gesteigerten Blutdruck wieder herabsetzt. Hitze und Kälte als solche rufen bei ihrer directen Einwirkung auf die Haut selten Jucken hervor. Dagegen rufen die Lichtstrablen der Sonne - allerdings indirect durch die Läsionen, die sie erzeugen - starken Pruritus hervor. Ja bei empfindlichen Leuten kann schon das diffuse Tageslicht intensiven urticariellen Pruritus an den exponirten Hautflächen hervorrufen.

Die einfache Reizbarkeit der Haut, die durch rauhe Unterkleider entsteht, ist therapeutisch von grosser Wichtigkeit.

III. A. S. Myrtle. Myrtle sprach über die Schwierigkeiten bei der Behandlung des Pruritus. Bei Fällen von Pruritus ani et vulvae hat er am wirksamsten den Gebrauch von Kali caust. fusum, gr. XV-Xl ad $3 \mathbf{j}$, gefunden.

IV. Henry Waldo führte aus, dass ein Gegenreiz für die vasomotorischen Centren mittelst Senfpflaster, das über dem Rückenmark angebracht würde, bei hartnäckigen Fällen zum Erfolg führe. Bei osteoarthritischem Pruritus sei natr. salicyl. allein oder in Verbindung mit Arsen nützlich.

V. G. G. Stopford Taylor bemerkte, dass constitutionelle Mittel in der Regel nicht viel nützten, obwohl man auf Symptome von Gicht, Rhematismus oder Dyspepsie immer sein Augenmerk richten müsste. Pruritus hiemalis würde am besten gemildert durch Bewegung und beruhigende Mittel, wie Glycerin-, Bleisalben etc. und baumwollene oder seidene Unterkleidung. Das tägliche Bad sollte untersagt werden. Bei Pruritus ani et scroti wäre die Anwendung spirituöser Mittel und die Trennung der aneinanderliegenden Hautfächen unerlässlich. Bäder mit schwachen Lösungen von Liq. carbon. deterg. wären ebenfalls für die Kranken sehr angenehm. Vortragender hat viele Fälle bei Radfahrern gesehen.

VI. F. H. Barendt betonte die Wichtigkeit localer Mittel, besonders bei den Fällen, wo sich nur Pruritus fände ohne Hautläsionen. Er lobte sehr die Anwendung von Blasenpflastern über der Gegend des Riückenmarkes, welche den Herd des Juckens versorgte. Unter vielen localen Mitteln hat sich ihm $2 \%$ heisses Carbololivenöl, Abends und Morgens je o Minuten fest eingerieben, an besten bewährt. 
VII. Elisabeth Garret Anderson legte dar, dass sie bei der Behandlung des nervösen Pruritus der Frauen gute Resultate erhalten habe, wenn sie die Menge der für die Patienten passenden Nahrung steigerte. Ruhe, besonders vor den Małlzeiten, wäre wichtig. Bäder wurden in diesen Fällen schlecht vertragen. Sie empfahl auch vollständig aus Seide bestehendes Unterzeug. Leberthran kann, wenn erforderlich, der Nahrung zugesetzt werden.

Nc Call Anderson hob im Schlussworte hervor, dass die Behandlung des ursächlichen Moments die Hauptsache wäre, er unterschätze jedoch nicht die locale Behandlung. Er glaube, dass der gute Einfluss von Harrogate z. B. nicht den Bädern dort zu verdanken sei, sondern der Diät und der gesunden Umgebung. Er stimme mit den Bemerkungen von Mrs. Anderson überein, dass es wichtig sei, die Ernährung hoch zu halten.

B. Pye-Smith, P. H. Affections of the skin oceurring in the course of Bright's disease.

Früher kannte man als einzige Hautstörung bei Bright'scher Krankheit das 1808 von Willan beschriebene Erythema leve. Es ist eine oberflächliche Dermatitis und hat mit den anderen Formen des echten Erythems nichts zu thun. Es gibt indessen noch andere Dermatitisformen, die nicht so selten im Verlaufe chronischer Bright'scher Krankheit auftreten: 1. Ein glänzend rother diffus Ausschlag, der hauptsächlich am Stamme auftritt, weniger ausgebreitet auf Nacken, Armen und Schenkeln und sehr selten im Gesicht, an Händen und Füssen. Eir unterscheidet sich durch den bei natürlichem oder künstlichem Schwitzen entstehenden Ausschlag, dem er etwas ähnlich sieht, durch die Localisation, durch das Fehlen von Sudamina und dass er ohne Anwendung heisser Bäder oder anderer Schwitzmittel entsteht, wenn die Haut hart und trocken ist. 2. Eine papulöse Eruption von grossen, einzeln stehenden ziemlich dunkelruthen Knötchen, die auf einer trockenen, rauhen, bisweilen schuppenden Oberfläche sitzen. Der Sitz ist gewöhnlich Aussenfläche der Schenkel und Beine, Schultern und Streckseite der Vorderarme, bisweilen auch Leisten und Abdomen. Vortragender sah die Affection nie im Gesicht oder an Händen und Füssen. 3. Fine feuchte Dermatitis, ähnlich dem Eczem, doch Arme und Beine crgreifend mit Freilassung der Gelenkbengen, des Gesichtes und der Ohren, ohne Reizerscheinungen und ohne dass sie je früher aufgetreten wäre. 4. Zweimal sah Vortragender eine sehr ausgebreitete und prophuse Dermatitis, die ganz der universellen exfoliativen Dermatitis Wils o n's ähnlich sah. - So verschieden diese 4 Formen sind, so haben sie doch manche Züge miteinander gemein. Sie sind oberflächlich und hinterlassen nach ihrem Verschwinden oder beim Tode keine Spur. Sie verlaufen acut oder subacut, nicht chronisch und recidiviren selten. Sie sind nie pustulös. Sie sind gelegentlich purpurfarben. Die subjectiven Symptome sind gewöhnlich leicht. Was die Prognose angeht, so treten sie gewöhnlich in den späteren Stadien der Nierenerkrankung auf und sind daher nicht selten 
vorhanden, wenn der Tod eintritt. Sie verschwinden aber auch oft lange vorher und dies bedeutet keine Versehlimmerung der übrigen Symptome. Was ihre Pathologie angeht, so bringt sie Verf. nicht mit Urämie in Zusammenhang. Eher kann man diese Hautentzündungen in Analogie bringen mit der Retinitis, den Entzündungen der serösen Häute und dem Lungenödem, von denen Nephritiker gern befallen werden. Was die Form der Nierenerkrankung angeht, bei der diese Hautaffectionen auftreten, so sah sie Vortragender nie bei acuter Nephritis mit Dedem noch bei Fettniere; entweder fanden sie sich in den späteren Stadien chronischer tubulärer Nephritis oder bei granulärer Degeneration mit Schrumpfung der Nierenriade. Die Behandlung ist nur local und besteht in Blei-, Zink- oder Wismuthsalben, bisweilen nur in Olivenöl oder Vaseline.

Radcliffe Crocker hob hervor, wie wichtig es sei, auf Formen von Dermatitis zu achten, die auf Erkrankung innerer Organe zurückzuführen seien. Seiner Erfahrung nach stimme er mit Dr. Pye-S mith über die Prognose nicht überein, da sie eine trübe sei bei diesen Fällen vorgeschrittener Bright'scher Krankheit.

Savill erwähnte mit Bezugnahme auf die von Pye-Smith erwähnten Fälle exfoliativer Dermatitis, dass ihn seine weitere Erfalırung über die „epidemische Hautkrankheit" dazn geführt habe anzunchmen, dass Nierenerkrankung für letztere eine Prädisposition schaffe. Von Patienten mit dieser Complication gingen alle - mit Ausnalıme von 5-6 Fällen - zu Grunde.

Bradbury hat mehrere Fälle von Hauterkrankung gegen das Ende des morbus Brightii auftreten gesehen. Er hat Erytheme beobachtet und einen Fall von Urticaria, Auch er betrachtet die Prognose dieser Fälle als ungünstig.

Pye-Smith erwähnte im Schlusswort nochmals, dass er nicht annehme, dass diese Erkrankungen auf Urämio beruhten; auch könne er die Prognose nicht als so schlecht betrachten. Urticaria sah er nie als Exanthemform.

C. Patrick Manson. On the Guinea-Worm.

Der reife Dracunculus Medinensis oder Guineawurm, erreicht eine Länge von 2,3 oder 4 Fuss. Dabei ist er sehr dünn, ungefähr $1 / 10$ engl. Zoll im Durchmesser. Die Anatomie des Thieres ist sehr einfach; man kann es beschreiben als einen musculo-cutanen Schlauch, der einen überaus dünnwandigen Uterus enthält, der sich vom Mund- bis zum Schwanzende erstreckt und mit Millionen aufgerollter, langgeschwänzter Embryonen angefüllt ist. Es ist nur das Weibchen bekannt, das Männchen kennt man noch nicht. Der Kopf des ausgewachsenen Wurmes ist abgerundet und ist mit einer punktförmigen Mundöffnung und einem Kranze kleiner Papillen versehen; das Schwanzende ist mit einer Art Haken versehen, die möglicherweise zum Festhalten dienen. Der Vordaungscanal ist ein kleiner Schlauch, der durch den Uterus zusammengedrückt und an eine Seite gedrängt ist. Vagina und Anus fehlen, oder man hat sio 
bisher nicht gesehen, wahrscheinlich sind sie durch den Druck des enormen Uterus obliterirt. Wenn die Embryonen im Uterus einen gewissen Reifegrad erlangt haben, so wandert der Wurm langsam durch das Bindegewebe seines Wirthes und zwar geht er stets abwärts, in die unteren Extremitäten. Gewöhnlich wandert er bis zum Knöchel oder Fuss. Hier bohrt er ein kleines Loch in das Derma; die darüber liegende Epidermis wird als eine Blase emporgehoben. Nach 1-2 Tagen platzt die Blase oder wird eingerissen, und es zeigt sich ein obcrflächliches Geschwür mit dem kleinen Loch, in dem der Kopf des Parasiten liegt. - Die vorherrschende Anschauung ist nun die, dass der Wurm jetzt herauskriecht oder ansgestossen wird, dass er dann der Auflösung anheimfällt und so die Limbryonen frei werden. Nach einer anderen Anschauung lässt der in die Gewebe eingedrungene Wurm die Jungen entschlüpfen mit der parnlenten Absonderung, die der Reiz der Anwesenheit des Thieres hervorruft. Beide Anschauungen sind indessen falsch: So lange der Wurm lebt, veranlasst er keine Eiterung und andererseits, sich selbst überlassen, verlässt, er den Körper nicht, bis er von seinen Jungen befreit ist. Vortragender konnte dies experimentell beweisen: Träufelt man mit einem Schwamm etwas kaltes Wasser auf die gesunde Haut, die das Guineawurmgeschwür umgibt, so sieht man aus dem erwähnten kleinen Loch nach wenigen Secunden ein Tröptchen einer weisslichen Flüssigkeit aufstejgen oder ein zarter Schlauch wird hervorgetrieben - einen Zoll weit oder mehr und bricht dann plötzlich auf. Sanmelt man etwas von der weisslichen Flässigkeit, die aufstieg oder aus dem zerplatzten. Schlauch entwich, so sieht man unter dem Mikroskop, dass sie Hunderte aufgerollter, anscheinend todter Embryonen des Guineawurmes enthält. Bringt man etwas Wasser unter das Deckglas, so sieht man nach wenigen Minuten, wie die Lmbryonen erwachen, sich strecken, sich bewegen und nach kurzer Zeit kräftig umherschwimmen. Sie sind offenbar in ihrem Element - im Wasser. - Die Erklärung dafür ist folgende: Der Guineawurm geht zum Fuss oder linöchel instinctiv, weil hier die Jungen am besten Gelegenheit finden, ins Wasser zu kommen. In Guineawurmgegenden tragen die Eingeborenen weder Schuhe noch Strümpfe, und ihre Füsse besonders kommen häufig in Wasserpfützen. Der Guineawurm lzennt diese Thatsache und empfindet es auch, wenn der Fuss seines Wirthes in Contact mit dem Wasser ist und entsendet dann, wie beim Experiment, seine Jungen. Er braucht ungefähr 14 Tage, um den Uterus völlig zu entleeren. Daun, wenn alle Jungen ausgeschlüpft sind, kommt er selbst heraus und kann dann mehr oder weniger leicht herausgezogen werden, oder er zerbricht und veranlasst Eiterung. Da der Parasit keine Vagina hat, entleert er die Jungen aus dem Munde. Die Contractionen der museulo-cutanen Wand lassen, wenn das auf die Haut des Wirthes wirkende Wasser den Reiz ausübt, den Uterus aus dem Munde des Thieres um 1-2 Zoll prolabiren, und dieses Stück bricht auf. Der Zweck all dieser seltsamen Anordnungen ist, dem Guineawurmembryo die beste Chance zu geben, als Zwischenwirth : :ine Süsswassercrustacee, cyclops quadricornis, zu erhalten, wie schon 
Fedschenko vor vielen Jahren fand. Dessen Experimente waren bisher nicht nachgepräft, und Vortragender hat dies gethan, da er im Seemannskrankenhause in Greenwich einen Fall von Guineawurm in Behandlung hatte. Er brachte Embryonen von Filaria mit diesen Cyclopiden zusammen und konnte die Metamorphose der ersteren Schritt für Schritt verfolgen. Nach dem Eindringen der Embryonen, die zu je 10-20 den Panzer zwischen den Gelenken der Bauchplatten durchdringen, verändern. sie ibre Gestalt. Während die freischwimmenden Embryonen von beiden. Seiten her abgeflacht sind, so werden sie, bald nachdem sie in den Cyclops eindrangen, cylindrisch. Nach einiger Zeit wirft der Embryo seine. quergestreifte Haut ab und zugleich damit seinen langen, dümen Schwimm schwanz, an dessen Stelle ein kurzer, konischer Schwanz, bisweilen nur ein abgerundeter Stumpf tritt. Offenbar hat $\mathrm{Fedschenko}$ diese Cylindrification des Embryo und die Bildung des conischen Schwanzes übersehen. Später mausert sich der Parasit nochmals; an Stelle des kurzen, conischen: Schwanzes tritt eine Art dreitheiligen Schwanzes, ähnlich einem kleinen, dreizähnigen Trepan. Gleichzeitig wird der junge Wurm grösser, sein Nahrungscanal verändert sich gewaltig und füllt sich schliesslich mit braunen, granulirten Massen an. Man muss annehmen, dass, wenn diese Metamorphose vollendet ist, der Parasit zur Uebertragung auf den Menschen durch Verschlucken mit dem Trinkwasser fertig ist, während er sich noch im Cyclops befindet. Vortragender sah die metamorphosirteit Guineawürmer im Cyclopskörper 70 Tage nach ihrem Eindringen leben. und sich bewegen. Neben den sehr interessanten biologischen Thatsachen enthalten diese Beobachtungen auch für Verhütung und Behandlung del Guineawurmkrankbeit einige Hinweise. Erstens zeigen sie, wie wichtig. es ist, in Filariagegenden nur filtrirtes oder gekochtes Trinkwasser zu geniessen. Zweitens lebren sie, dass es verkehrt ist, einen Guineawurm auszuziehen, bevor er alle Jungen entleert hat, da anderenfalls das Thier zerreisst; Myriaden von Jungen in die Gewebe entleert und so Zellgewebsentzündung und starke Eiterung entsteht. Versuchen kann man, wie neuerdings empfohlen, local schwache Sublimatlösungen zu injiciren, so das Thier zu tödten und dessen Absorption unter aseptischen Bedingungen zu ermöglichen.

Harrison frug Manson, ob es möglich wäre, dass eine Masse von Guineawürmern sich in der Pleurahöhle einer $R$ hea (des amerikanischen Strausses) einkapseln könnten. da er vor 2 Jahren bei einem solchen Thiere eine grosse Cyste mit 30-40 Würmern fand, die den Guineawürmern sehr ähnlich sahen.

Manson erwiderte, dass bei Hunden und vielen anderen Thieren verschiedene Formen von Filarien und anderen Würmern in den Lungengefässen angetrofien worden seien. Es sei bekannt, dass der Guineawurm bei vielen Thieren - Hunden, Pferden etc. - auftrete; ob er aber in. den Lungen gefunden worden sei, wisse er nicht.

v. Inscusion on diet in the etiology and treatment of diseases of the skin. 
I. W. Allan Jamieson. Einige Nahrungsmittel rufeu Hautstörungen unmittelbar hervor, indem sie toxisch wirken. Hierfür ist die Urticaria das beste Beispiel. Häufiger ruft zu lanosame und langdanernde schlechte Assimilation Hautkrankheiten hervor d. h. sie prädisponirt dazu und irgend eine Zufälligkeit lässt sie dann zur Entwicklung kommen. So ist bei der Pellagra der verdorbene Mais das eigentliche Mittel, das den Körper krankhaft stört, während die Sonnenstrahlen die Hautmanifestation, das Erythem, hervorrufen. Vielleicht entstehen auf ähnliche Weise Xeroderma pigmentosum und hydroa vaceiniforme.

Alkoholmissbrauch erzeugt einerseits den klebrig feuchten Schweiss, der sich an den Händen des Gewohnheitstrinkers zeigt, und andererseits eine starke und permanente Dilatation der Blutgefässe des Gesichtes und ruft so Rosacea hervor und eine grobe, fettige Haut. Da dies aber nicht bei allen Leuten eintritt, die diesem Laster huldigen, so müssen wir vielfach einen erregenden Factor heranziehen: Einer von diesen ist sicherlich: jeder Witterung ausgesetzt zu sein, andere: Einsperren in enge, schlecht ventilirte Räume und anhaltendes Bücken. -- Thee, in zu grossen Mengen, zu stark oder zu heiss genossen, ruft kalte Hände und Füsse hervor, wahrscheinlich in Combination mit chronischem, subacutem Gastro-intestinalcatarrh. Nach White sollen Früchte, die viel Säure enthalten, acute Eezeme hervorbringen. - Der beständige Mangel frischer grüner Gemüse, Salate, reifer Früchte führt zu einer Schwäche und Erschlaffung der Gewebe mit Alteration der Blutgefässe und der Zusammensetzung des Blutes selbst, in Folge deren Blutergüsse in die Haut eintreten und ein Zustand der Haut, dessen höchste Entwicklungsstufe wir "Scorbut" nennen. Bisweilen ruft die Muttermilch ähnliche Zustände, wenn auch milderer Art, hervor, wenn ihr gewisse Salze fehlen. Vortragender zeigt an verschiedenen Krankheitstypen: Eczem, Alopecia areata, Psoriasis etc., wie seiner Meinung nach Fehler in der Ernährung diese Krankheiten hervorrufen, resp. sie unterstützen und die Heilung aufhalten. Er geht dann über zu der "Diät bei der Behandlung der Hautkrankheiten", die im wesentlichen eine individualisirende sein muss.

IL. Smith, Walter G. fasst seine Austührungen folgendermassen zusammen.

1. Nur sehr wenige Hauterkrankungen können direct auf Fehler in der Diät zurückgeführt werden, wohl aber kann eine ungeeignete Diät schon vorhandene Eruptionen verschlimmern. Idyosinkrasie muss man als Ursache zulassen.

2. Die Krankheiten, die so entstehen, sind transitorischen Charakters und gehören meistens zu den Erythernen.

3. Diät hat nur geringen Einfluss auf die Heilung von Hautkrankheiten. Die Resultate bleiben weit hinter den landläufigen Erwartungen zurück, selbst bei Krankheiten wie Acne rosacea, wo wir uns verleiten lassen, viel von ihr zu hoffen.

4. Vermeidung von Alkohol, Regulirung der Verdauung und Beseitigung von Anämie sind von unendlich grösserem Einfluss als specielle Diätvorschriften. 
E. Pernet, George. The etiology of acute pemphigus. Pernet kommt in seiner Arbeit zu folgenden Schlüssen: 1. Es gribt eine Gruppe seltener Fälle acuter bullösel Eruptionen, die mit schweren Allgemeinerscheinungen einhergehen, gewöhnlich tödtlich enden und Fleischer befallen. 2. Die Krankheit tritt auf nach einer Wunde an den Händen oder Fingern. 3. Sie entsteht wahrseheinlich dureh einen Mikroorganismus. 4. Dieselben ätiologischen Factoren sind wahrscheinJich bei einer anderen Gruppe ähnlicher Fälle wirksam, bei denen die Patienten durch ihre Beschäftigung in Contact mit Thieren oder Theilen todter 'Thiere z. B. mit Fleisch oder Häuten gebracht werden. All diese Punkte crfordern noch weitere Nachforschung. In Zukunft würde es gut sein, wenn in allen zur Beobachtung kommenden Fällen auf folgende Data geachtet würde. 1. Beschäftigung a) Fleischer, b) Köche, Gerber, Nelker etc. 2. Nachforschung, ob eine Wunde vorhanden war. 3. Vorhandensein oder Fehlen eines charakteristischen Mikroorganismus, nämlich des Demme'schen Diplococcus. 4. Contact mit Thieren, die an einem bullösen oder vesiculösen Ausschlag litten. - Vortragender macht zum Schluss darauf aufmerksam, wie wichtig es ist, Fingerwunden, Saumgeschwüre etc. bei Fleischern und Leuten. die mit Fleisch zu thun haben, auf das gründlichste mit starken Antisepticis zu desinficiren.

F. Roberts, Leslie. The treatment of trichophytosis (ringworn) based on the physiology of the trichophytons.

Leslie Roberts gibt als Resultat seiner Arbeit Folgendes: 1. Es gibt in der Pflanzenwelt eine Classe kleiner, blütbenloser Pflanzen (fungi), die im Stande sind, Haar und andere Horngewebe zu zersetzen und zu assimiliren. 2. Dieser Process kann trichophytosis genannt werden und hat einige Varietaten. 3. Dieser Process kann stattfinden anf der Haut des lebenden Wirthes oder in dem Haar, das keine Beziehungen mit dem lebenden Organismus hat. 4. Trichophytosis follicularis ist die Combination der saprophytischen Zerstörung eines corpus iners (des Haares, Ref.) mit pathologischen Zellprocessen. 5. Ist erst einmal der Pilz in das Innere des Follikels eingedrungen, so ist jede Möglichkeit, ihm direct Tinbalt gebieten zu können, verloren. 6. Unser therapeutischer Einfluss auf den Pilz berubt darauf, dass wir im Stande sind, die weichen Zellen des Follikels zur Proliferation anzuregen. 7. In vielen liällen ist es wünschenswerth, diese Anregung über die physiologischen Grenzen hinaus zu steigern, so dass Eiter gebildet wird. 8. Tpitheliale Eiterbildung hat Jxeinen merkbaren Gewebsverlust zur Folge, während Bindegewebseiterung anabwendbar zu Verschwärung führt. 9. Wir müssen uns daher bemühen, die Eiterung auf das Epithel zu beschränken.

G. Anderson, William. A case of adenoma sebaceum, intormingled with flaceid mollusca fibrosa: treatment by excisjon.

In diesem Falle wurde cin sehr gutes Resultat erzielt. Vortr. empfiehlt boi solchen Fällen sorgfältig und bedacht mit dem Messer vor:zugehen. 
H. Waido, Henry. Alopecia a reata.

Waldo bält, vom klinischen Standpunkt aus, die grosse Mehrzahl der Fälle von Alopecia areata nicht für parasitären Ursprungs. Die häufigsten Ursachen sind Ueberarbeitung, zu wenig Schlaf, schlechte hygienische Verhältnisse und, vielleicht, dazukommende schlechte Ernährung. Er erhiolt die besten Resultate durch Besserung des Allgemeinzustandes der Patienten conbinirt mut mässiger Stimulation der mehr oder weniger anästhetischen Flächen.

I. Stowers, J. H. A case of dermatitis repens.

Die einzelnen Symptome der Krankheit ergetben, dass er sich um eine distincte Affection handelt, obwohl sie in einzelnen ihrer Charaktere dem Eczem sehr ähnelt. Bei seinem Falle wies der Vortr. auf folgende Punkte hin: 1. Das Alter und die Umstände, unter denen die Krankheit begann, 14 Tage nach dem Wochenbett. 2. Ihre Hartnäckigkeit und lange Daner, nämlich 44 Jahre. 3. Das Befallensein beider Füsse und beider Hände. 4. Die Störungen des Nervensystems. 5. Das Vorhandensein deutlicher "glossy skin". 6. Die Verbindung mit recurrirender acuter Gicht. 7. Das Fehlen von Verletzungen und der wahrscheinlich idiopathische Ursprung der Krankheit.

K. Colcott Fox. An uncommon papulo-pustularexuption in infants.

Die Affection war nicht syphilitischen Ursprungs. Vortr. betrachtet die Eruption nicht direct als tuberculös, glaubt aber, dass sie bei Kindern auftritt, die schlecht ernährt und zur Infection mit Tuberculose geneigt sind. Bei solchen Kindern findet ein Organismus in den Follike]n einen günstigen Nährboden. Er ist geneigt, die Affection einer anderen zuzurechnen, die jetzt in Jundon als Acne scrofulosorum wohl bekannt ist.

L. Smith, Gilbert. A case of lichen planus verrucosus.

Smith nimmt als Ursache in seinem Falle nervöse Erschöpfung bei einer nervösen Frau an und zwar durch Störung der Generationsorgane. Der Fall war auch interessant durch den Umfang der Affection, mehr aber noch durch die Thatsache, dass die warzigen Flecken sich ableiten liessen von den konischen Knötchen, die an den Haarfollikeln sassen und nicht von den platten Knötchen. Sonst ist der Process derselbe wie bei Lichen planus.

M. Mackey, Edward. Two cases of cheiro-pompholyx in association with eczema.

Vortr. ist der Ansicht, dass die Beziehung zwischen Cheiro-Pompholyx und Eczem eine engere ist als gewöhnlich angenommen wird; ferner, dass die Pathologie beider nicht sehr verschieden ist. Er glaubt, dass Cheiro-Pompholyx eine Varietät des Eczems ist.

N. Barendt, H. A case of cheiro-pompholyx.

Barend betrachtet den Cheiro-Pompholyx als eine Neurose, d. h. als eine Störung der Vasomotoren der Finger.

0. Eddowes, Alfred. Corns, true and so-called. 
Eddowes bemerkt, dass oft Warzen auf den Fussoblen oder an der Ferse dem äusseren Ansehen nach Hühnerangen glichen und für solche gehalten würden. In solchen Fällen nützen die Massregeln, die bei Hühneraugen wirksam sind, als Veränderungen des Schuhzeuges oder erweichende Pflaster nichts, was von praktischer Bedeutung ist. Die Behandlung muss natürlich die für Warzen passende sein.

P. Galloway. The skin lesions of syringomyelia.

Galloway sagte in seinem Vortrage, dass die klinisehen Erscheinungen die experimentellen Resultate von Mott, Sherrington, Ferrier und $T$ un er bestätigten, dass nämlich sensorische Impulse durch die graue Masse geleitet würden und nicht, wie man früher behauptete, durch die aufsteigende Vorder-Seitenstrang- oder Hinterstrangbahn. Die zahlreichen Hautlaesionen, die eine so wichtige Rolle spielen bei dem Morvan'schen Typus der Krankheit werden durch locale, infectiöse Processe hervorgerufen. Möglich ist auch eine ascendirende, toxische Neuritis ausgehend von dem erkrankten Theil oder veranlasst durch Absorption bacterieller Gifte.

Q. Harrison, J. A. Two cases of unusual verruca necrogenica. Es handelte sich in den beiden, gegen die Behandlung sehr widerspenstigen Fällen weder um eigentliche verruca necrogenica noch Lupus, sondern um Hauttuberculose. Bacillen wurden nicht gefunden.

R. Liddell, J. A case of pityriasis rubra pilaris (I)evergie): Clinical features and minute anatomy.

Lid dell betrachtet die Veränderungen an Fpidermis, Haarfollikeln, Schweissdrüsen und Talgdrüsen als primär und nicht als eine Folge von Veränderungen im Corium. Ob die Reizquelle für die erzeugten Veränderungen innerhalb des Körpers oder ausserhalb zu suchen sei, bleibt eine offene Frage. Davon ausgehend, dass die Läsionen symmetrisch vertheilt seien, kann man geneigt sein, den Zustand als Trophoneurose aufzufassen; andererseits kann auch der Reiz von aussen kornmen. Vortr. fand einen Parasiten in Schnitten excidirter Haut, in welchen Beziehungen dieser aber zur Krankheit steht, ist noch nicht entschieden.

Alfred Sternthal (Braunschweig). 